Chirurg 2021 · 92:1123-1131

https://doi.org/10.1007/s00104-021-01403-y

Angenommen: 27. Februar 2021

Online publiziert: 14. April 2021

๑ Der/die Autor(en) 2021

\author{
Jasmin Dillner ${ }^{1}$ Frank Meyer ${ }^{1}$ ' Zuhir Halloul' $\cdot$ Michael Görtler ${ }^{2}$ \\ 'Klinik für Allgemein-, Viszeral-, Gefäß- und Transplantationschirurgie, Universitätsklinikum Magdeburg \\ A.ö.R., Magdeburg, Deutschland \\ ${ }^{2}$ Klinik für Neurologie, Universitätsklinikum Magdeburg A.ö.R., Magdeburg, Deutschland
}

\title{
Intraoperative Angiographie bei A.-carotis-Rekonstruktion - pathologische Befunde, Zuverlässigkeit und Bedeutung des Verfahrens
}

welche technischen Unzulänglichkeiten relevant und korrekturbedürftig sind $[5,13,24]$. Weitere mögliche Gründe könnten eine zu geringe Sensitivität der intraoperativen Angiographie für einen pathologischen Befund sein und/oder eine nicht ausreichende Sensitivität der Ad-hoc-Beurteilung durch den Operateur $[5,18]$.

Ziel der hier vorgestellten Studie war, die beiden letztgenannten Aspekte zu untersuchen und zu quantifizieren. Dazu wurden (1) intraoperativ durchgeführte Angiographien von einem unabhängigen Gefäßchirurgen nachbeurteilt sowie (2) ein prospektiv 3 bis 5 Tage postoperativ erhobener duplexsonographischer Befund mit der angiographischen Nachbeurteilung verglichen. Darüber hinaus wurden klinische und operative Variablen auf ihre Assoziation mit operativtechnischen Unzulänglichkeiten überprüft.

\section{Patienten, Material und Methoden}

\section{Patientenauswahl}

Alle Patienten, die sich von 1995 bis 2012 in der Abteilung Gefäßchirurgie der Universitätsklinik für Allgemein-, Viszeral-, Gefäß- und Transplantationschirurgie am Universitätsklinikum Magdeburg A.ö.R. der TEA einer A.-carotis-Stenose unterzogen und bei denen im Anschluss an die Rekonstruktion intraoperativ eine angiographische Kontrolle erfolgte, wurden für diese Studie ausgewählt. Seit 2004 wurde diese Kontrolle routinemäßig bei jeder TEA durchgeführt, davor war dies nur sporadisch der Fall. Alle diagnostischen und therapeutischen Maßnahmen erfolgten im Rahmen des klinischen Behandlungsstandards, die Datendokumentation und -speicherung fand nach den Regeln nationaler und internationaler Datenschutzverordnungen statt. Die zuständige Ethikkommission der Ottovon-Guericke-Universität Magdeburg verneinte daher die Notwendigkeit einer Patientenzustimmung für die retrospektive Datenauswertung. Die Studie wurde im Deutschen Register Klinischer Studien (DRKS) unter der Studiennummer DRKS00023151 registriert.

\section{Präoperative Untersuchungen}

Die standardisierte präoperative Diagnostik umfasste die Anamnese, den neurologischen Status, ein zerebrales Computertomogramm (CT)/Magnetresonanztomogramm (MRT), Laboruntersuchungen und ein Elektrokardiogramm (EKG). Der extra- und intrakranielle Gefäßstatus wurde mittels Doppler- und farbkodierter Duplexsonographie erhoben. Im Fall eines unklaren sonographischen Befundes oder eines fehlenden 
temporalen Schallfensters zur Beurteilung der intrakraniellen Gefäße erfolgte zusätzlich eine MR- oder CT-Angiographie. Der Stenosegrad der A. carotis interna (ACI) wurde duplexsonographisch nach publizierten Kriterien bestimmt [1] und als distale Durchmesserreduktion (NASCET [North American Symptomatic Carotid Endarterectomy Trial]Stenosegrad) in $10 \%$-Schritten angegeben. Bei Patienten mit einem temporalen Schallfenster wurde zusätzlich die zerebrovaskuläre Reservekapazität mittels Doppler- $\mathrm{CO}_{2}$-Test bestimmt $[8,21]$.

\section{Anästhesie- und Operations- verfahren}

Die TEA wurde routinemäßig als PatchEndarteriektomie intendiert und erfolgte bis 2001 in Allgemeinanästhesie unter Einsatz eines Shunts. Seit 2002 kam eine Regionalanästhesie mit kombinierter superfizieller und tiefer zervikaler Plexusanästhesie zur Anwendung. Vor dem Abklemmen der A. carotis wurde Heparin intravenös appliziert und am Operationsende mittels Protaminsulphat in Höhe der halben applizierten Heparindosis antagonisiert. Bei der Operation in Regionalanästhesie wurde die Notwendigkeit eines Shunts klinisch überprüft (Drücken einer „Quietsch-Ente“ mit der Hand kontralateral zur Operationsseite). Im Weiteren erfolgten die Längsarteriotomie und die direkte Desobliteration. Bei sichtbarer Intimastufe nach distal wurde diese fixiert und im Anschluss die Längsarteriotomie mittels Patchplastik verschlossen. Nach Beendigung der Patchnaht erfolgte die intraoperative Angiographiekontrolle durch einen C-Bogen (OEC 9600/9800, General Electric; seit 2009 Arcadis Avantic, Siemens, München, Deutschland) mittels Punktion der A. carotis communis (ACC) proximal des eingenähten Patches und Injektion von 5-10 ml jodhaltigem Kontrastmittel (Imeron 300, Bracco) über eine 22-Gauche-Butterfly-Kanüle. Standardmäßig erfolgte eine Aufnahme in $30^{\circ}$-lateraler Projektion, bei unklarer Situation ggf. erweitert um eine a.p. oder laterale Projektion bzw. nochmalige Injektion. Bei unauffälligen Perfusionsverhältnissen im Patchbereich und in der Abstrombahn wurde die Operation beendet. Postoperativ wurde der Patient für $24 \mathrm{~h}$ auf der Stroke-Unit überwacht.

\section{Intraoperative DSA und postoperative Duplexsonographie}

Die intraoperative Angiographie wurde vom Operateur vorgenommen und in Form eines oder mehrerer Bilder dokumentiert (Print, seit 2009 digital). Die Beurteilung erfolgte durch ihn bzw., im Fall eines weniger erfahrenen Chirurgen, durch den in derartigen Fällen mitoperierenden und supervidierenden Gefäßchirurgen. Als Indikation für einen chirurgischen Korrektureingriff galten pathologische Befunde i.S. von Stenosen $>60 \%$ und ein Verschluss im Bereich der endarteriektomierten ACC und ACI. Neu eingetretene Verschlüsse nur in der Abstrombahn stellten eine Indikation für eine unmittelbar postoperative endovaskuläre Thrombektomie dar. Korrektureingriffe bei ACE-Pathologien wurden vom Operateur entschieden, für hochgradige Stenosen in der Abstrombahn erfolgte dies ad hoc gemeinsam gefäßchirurgisch und neurologisch. Die 3 bis 5 Tage postoperativ - bei (neuer) postoperativer neurologischer Symptomatik umgehend - durchgeführte Duplexsonographie erfolgte durch einen DEGUMzertifizierten Neurologen, dem die angiographischen Befunde und ein möglicher Korrektureingriff nicht bekannt waren. Lokalbefund (ACC; ACI; A. carotis externa, ACE) und Abstrombahn (extrakranielle distale und intrakranielle ACI; A. cerebri media, ACM) wurden duplexsonographisch jeweils getrennt als pathologisch/nichtpathologisch beurteilt. Im pathologischen Fall erfolgte eine Quantifizierung des Ausmaßes des pathologischen Befundes (Verschluss, hochgradige Stenose, nicht hochgradig stenosierend) und eine Einschätzung der (vermeintlichen) Ätiologie (Thrombus/ Thrombembolie, Nahteinziehung, Intima-Media-Ablösung, langstreckig enges Gefäß, residualer Plaque, Kalibersprung/ Kinking am Übergang des Patches zur distalen ACI, extravasales Hämatom). Die Zweitbeurteilung der dokumentierten digitalen Subtraktionsangiographien wurde durch einen gefäßchirurgischen
Facharzt mit TEA-Erfahrung retrospektiv im Anschluss an den Patienteneinschlusszeitraum vorgenommen, jeweils ohne Kenntnis der intraoperativen Befundung, einer möglicherweise stattgefundenen Korrekturoperation und des postoperativen duplexsonographischen Befundes. Die angiographische Zweitbeurteilung erfolgte analog zur Duplexsonographie.

\section{Klinisch-neurologisches Outcome}

Bei einer ambulanten Wiedervorstellung des Patienten 6 Wochen postoperativ wurden mögliche Komplikationen, die erst poststationär aufgetreten waren, erfragt und der klinisch-neurologische Status erhoben. Patienten, die diesen Termin nicht wahrnahmen, wurden telefonisch kontaktiert. Die bis 4 Wochen postoperativ eingetretenen Befunde gingen in das perioperative klinischneurologische Outcome (persistierender Schlaganfall und Tod) ein.

\section{Vergleich der Untersuchungs- verfahren und Prädiktoren für intraoperative Pathologika}

Die Sensitivität der prospektiven und retrospektiven angiographischen Beurteilung und der prospektiven duplexsonographischen Untersuchung wurde für (1) Verschluss und hochgradige Stenose lokal im Operationsbereich (ohne ACEPathologie) und in der Abstrombahn sowie (2) jedwede Pathologie lokal im Operationsbereich (ohne ACE-Pathologien) untersucht. In einem zweiten Schritt wurde untersucht, ob klinische und operationstechnische Variablen mit dem Auftreten dieser operativen Pathologika assoziiert waren und inwieweit das Auftreten derartiger operativer Pathologika mit dem klinisch-neurologischen Outcome assoziiert war.

\section{Statistik}

Die statistische Auswertung erfolgte mittels SPSS, Version 26 (SPSS Inc., Chicago/IL, USA). Der Vergleich zwischen den Untersuchungsmethoden erfolgte für die beiden angiographischen Auswertungen paarweise mittels McNemar- 
Chirurg 2021 · 92:1123-1131 https://doi.org/10.1007/s00104-021-01403-y

(c) Der/die Autor(en) 2021

\section{J. Dillner · F. Meyer · Z. Halloul · M. Görtler}

\section{Intraoperative Angiographie bei A.-carotis-Rekonstruktion - pathologische Befunde, Zuverlässigkeit und Bedeutung des Verfahrens}

\section{Zusammenfassung}

Ziel. Der Wert der intraoperativen Angiographie und deren Ad-hoc-Beurteilung im Hinblick auf operationstechnische Unzulänglichkeiten soll geprüft werden. Methode. Insgesamt 523 konsekutive A. cartotis-Thrombendarteriektomie(TEA)Patienten mit intraoperativer Kontrollangiographie, postoperativer Duplexsonographie und retrospektiver Zweitbeurteilung der Angiographie wurden in die Auswertung einbezogen.

Ergebnisse. In der retrospektiven Zweitbeurteilung der Angiographie wurden $23(4,4 \%)$ Verschlüsse oder hochgradige Stenosen der Arteria carotis communis (ACC) oder Arteria carotis interna (ACl) im Operationsbereich (12/2,3\%) bzw. in der Abstrombahn (distale extrakranielle und intrakranielle $\mathrm{ACl}, \mathrm{A}$. cerebri media $[\mathrm{ACM}]$ ) detektiert (11/2,1\%), wohingegen bei der intraoperativen Ad-hoc-Beurteilung nur 13 $(2,5 \%)$ derartige Pathologien beschrieben worden waren ( $7 / 1,3 \%$ im Operationsbereich, $6 / 1,1 \%$ in der Abstrombahn; $p=0,002$ ). Duplexsonographisch wurden postoperativ bei 50 von 505 untersuchten Patienten (10,1\%) operationstechnische Unzulänglichkeiten lokal detektiert, was signifikant mehr war als in der Angiographie $(p<0,001)$. In den meisten Fällen handelte es sich um nicht okkludierende/nicht hochgradig stenosierende IntimaMedia-Ablösungen (19), Nahteinziehungen (13) und Kinkings/Kalibersprünge am distalen Patchende (14). Nahteinziehungen und Kinkings/Kalibersprünge waren mit einer linksseitigen TEA (adjustierte OR: 2,4; 95\%KI: 1,1-5,1), einer Operation ohne Patch (adjustierte OR: 16,6; 95\%-KI: 1,3-215,0) und der Verwendung eines Dacron- vs. Polytetrafluorethylen-Patch (adjustierte OR: 3,0; 95\%-KI: 1,4-6,6) assoziiert.

Schlussfolgerung. Bei der Ad-hoc-Beurteilung der intraoperativen Kontrollangiographie kann eine nicht unerhebliche Zahl auch okkludierender und hochgradig stenosierender Pathologien übersehen werden (zur Detektion nicht okkludierender und nicht hochgradig stenosierender operativer Unzulänglichkeiten methodisch nicht geeignet - Ausweich: postoperative Duplexsonographie).

Schlüsselwörter

A.-carotis-Desobliteration - Duplexsonographie - Postoperatives Komplikationsprofil · Outcome $\cdot$ Morbidität

\section{Intraoperative angiography in carotid artery reconstruction-Pathological findings, reliability and importance of the procedure}

\section{Abstract}

Aim. To investigate the value of intraoperative angiography and its ad hoc evaluation with respect to cases of surgical technical inaccessibility.

Methods. Overall, 523 consecutive carotid artery thrombendarterectomy (TEA) patients with intraoperative control angiography, postoperative color-coded duplex sonography and retrospective re-evaluation of documented angiographic images were included in the evaluation.

Results. In the retrospective angiographic re-evaluation 23 (4.4\%) occlusions or highgrade stenoses of the common carotid artery (CCA) or internal carotid artery (ICA) in the surgical field $(12,2.3 \%)$ or of downstream ICA or middle cerebral artery (MCA, 11, $2.1 \%$ ) were detected. The detection rate was significantly lower in the intraoperative ad hoc evaluation with overall only 13 (2.5\%) detected pathologies $(7,1.3 \%$ in the surgical field, $6,1.1 \%$ in large downstream arteries, $p=0.002$ ). Postoperative duplex sonography performed in 505 patients detected 50 cases $(10.1 \%)$ of local surgical technical inaccessibility, which was significantly more than in the angiography $(p<0.001)$. In most cases these were nonocclusive, low-grade stenosing detachments of the intima media $(n=19), 13$ suture contractions, and 14 kinking/abrupt diameter changes at the distal end of the patch. Suture contractions and kinking/diameter changes were associated with a left-sided TEA (adjusted odds ratio, OR 2.4, 95\% confidence interval, Cl 1.1-5.1), an operation without a patch (adjusted OR, 16.6,
95\% Cl 1.3-215.0), and using Dacron patches in contrast to PTFE patches (adjusted OR 3.0, 95\% Cl 1.4-6.6).

Conclusion. The ad hoc evaluation of intraoperative completion angiography by surgeons missed a substantial number also of occluding and severely stenosing pathologies. Angiography is not suitable for the detection of nonocclusive and low-grade stenosing cases of operative inaccessibility. Postoperative color-coded duplex sonography is an adequate tool for surgical quality control.

Keywords

Carotid artery occlusion - Duplex ultrasonography - Postoperative complication profile. Outcome $\cdot$ Morbidity
Test und bei Einbeziehung zusätzlich der Duplexsonographie mittels Cochran-QTest jeweils für verbundene Stichproben. Eine mögliche Assoziation zwischen klinischen und operationstechnischen Variablen und dem Auftreten operativer Pathologien sowie zwischen Letzteren und dem Auftreten eines perioperativen Schlaganfalls/Todes wurde mittels mul- tivariater binärer logistischer Regression untersucht. Dabei wurden die unabhängigen Input-Variablen initial eingeschlossen und danach schrittweise rückwärts aus der Regression ausgeschlos$\operatorname{sen}\left(p_{\text {Ausschluss }} \geq 0,1 ; p_{\text {Wiedereinschluss }}<0,05\right)$. Das Ergebnis für die in der Regressionsanalyse verbliebenen Variable wird als adjustierte Odds Ratio (OR) mit 95\%-
Konfidenzintervall (KI) präsentiert. Als statistisch signifikant wurde eine Irrtumswahrscheinlichkeit von $p<0,05$ (zweiseitig) festgelegt.

\section{Ergebnisse}

Präoperativ waren 294 der 523 operierten Stenosen $(56,2 \%)$ symptomatisch. 
Tab. 1 Patientencharakteristika, klinische und operative Daten von 523 TEA-Patienten mit intraoperativer Kontroll-DSA (digitale Subtraktionsangiographie)

\begin{tabular}{|c|c|}
\hline Demographische, klinische und operative Daten & $n(\%)$ \\
\hline Alter (Mittelwert [Standardabweichung], Jahre) & $67,8(9,5)$ \\
\hline Männer & $386(73,8)$ \\
\hline Arterielle Hypertonie & $468(89,5)$ \\
\hline Diabetes mellitus & $199(36,0)$ \\
\hline Koronare Herzkrankheit & $183(35,0)$ \\
\hline \multicolumn{2}{|l|}{ Symptomatik: } \\
\hline Asymptomatisch & $229(43,8)$ \\
\hline Amaurosis fugax & $50(9,6)$ \\
\hline TIA & $57(10,9)$ \\
\hline Schlaganfall & $187(35,8)$ \\
\hline \multicolumn{2}{|l|}{ Stenosegrad (NASCET): } \\
\hline $50 \%$ & $6(1,1)$ \\
\hline $60 \%$ & $14(2,7)$ \\
\hline $70 \%$ & $174(33,3)$ \\
\hline $80 \%$ & $202(38,6)$ \\
\hline $90 \%$ & $60(11,5)$ \\
\hline$>90 \%$ & $58(11,1)$ \\
\hline $100 \%$ & $9(1,7)$ \\
\hline Kontralateraler Verschluss/hochgradige Stenose & $111(21,2)$ \\
\hline Aufgehobene zerebrovaskuläre Reservekapazität & $43(8,2)$ \\
\hline Regionalanästhesie (vs. Allgemeinanästhesie) & $443(84,7)$ \\
\hline TEA-Seite rechts & $253(48,4)$ \\
\hline Rechtshändigkeit des Erstoperateurs & $523(100)$ \\
\hline \multicolumn{2}{|l|}{ TEA-Frequenz Erstoperateur } \\
\hline$<20$ Operationen & $67(12,8)$ \\
\hline$<50$ Operationen & $42(8,0)$ \\
\hline$\geq 50$ Operationen & $111(21,2)$ \\
\hline$\geq 50$ Operationen und $\geq 20$ Operationen/Jahr & $303(57,9)$ \\
\hline Shunt & $142(27,2)$ \\
\hline Intimafixierung distal & $480(91,8)$ \\
\hline \multicolumn{2}{|l|}{ Präoperative TAH } \\
\hline Keine TAH & $64(12,2)$ \\
\hline $1 \mathrm{TAH}$ & $353(67,5)$ \\
\hline $2 \mathrm{TAH}$ & $106(20,3)$ \\
\hline \multicolumn{2}{|c|}{$\begin{array}{l}\text { TEA Thrombendarteriektomie, DSA digitale Subtraktionsangiopathie, TIA transitorische ischämisch } \\
\text { Attacke, TAH Thrombozytenaggregationshemmer, NASCET North American Symptomatic Carotid } \\
\text { Endarterectomy Trial }\end{array}$} \\
\hline
\end{tabular}

Bei 9 Patienten (1,7\%) bestand ein kurzstreckiger segmentaler Verschluss von der Bifurkation bis zum Abgang einer primitiven karotidovertebralen Anastomose. Bei 512 TEA handelte es sich um eine prophylaktische Erst- (509) oder Reoperation (3), bei 12 Patienten erfolgte der Eingriff im Rahmen eines akuten Schlaganfalls aufgrund einer zerebral-hämodynamischen Minderperfusion. 520 Stenosen (99,4\%) wurden in Form einer Patch-Endarte- riektomie operiert, 2-mal erfolgte eine Eversionsendarteriektomie, 1-mal eine Direktnaht. Als Patchmaterial kamen bei 215 Operationen Dacron $(41,1 \%)$, bei 296 Polytetrafluoethylen (PTFE; 56,6\%), bei 8 bovines Perikard (1,5\%) und bei einer TEA eine autologe Vene zum Einsatz. Weitere Patientencharakteristika, klinische und Operationsdaten sind in - Tab. 1 aufgeführt.

Perioperativ erlitten 21 Patienten $(4,0 \%)$ einen Schlaganfall, wovon 4 letal verliefen. Drei weitere Patienten starben 2, 9 bzw. 14 Tage postoperativ an einem Myokardinfarkt, ein Patient 3 Wochen nach der TEA an einer Pneumonie.

\section{Intraoperative DSA und Sofortkorrekturen}

Intraoperativ wurden 21 Angiographien $(4,0 \%)$ als pathologisch beurteilt. Bei den 15 lokalen Pathologien (2,9\%) handelte es sich um 6 hochgradige Stenosen (1 in ACC, 5 in ACI) und 9 Verschlüsse ( 1 in ACI, 8 in ACE), von denen alle 6 hochgradige Stenosen, der ACIVerschluss und 5 ACE-Verschlüsse korrigiert wurden (Sofortkorrekturrate lokal: 2,3\%). In der Abstrombahn wurden 3 Verschlüsse und 3 hochgradige Stenosen $(1,1 \%)$ neu gegenüber präoperativ beschrieben, von denen alle 3 Verschlüsse unmittelbar postoperativ endovaskulär thrombektomiert wurden. Die Ätiologie der 6 hochgradigen Stenosen im Operationsbereich war jeweils eine Nahteinziehung, die des lokalen ACIVerschlusses und der 6 Pathologien in der Abstrombahn eine Thrombose bzw. Thrombembolie. ACE-Pathologien wurde keine Ätiologie zugeordnet, dies war anhand der Angiographie nicht ausreichend valide möglich (• Tab. 2).

Keiner der 15 Patienten mit einer lokalen Pathologie bot postoperativ eine (neue) neurologische Symptomatik oder hatte, sofern in Regionalanästhesie operiert (1 ACC-Stenose, 1 ACI-Verschluss, 3 hochgradige ACI-Stenosen, 8 ACEVerschlüsse), intraoperativ eine Symptomatikgeboten. Keiner der 3 Patienten mit einer hochgradigen Stenose in der Abstrombahn - jeweils in Regionalanästhesie operiert - hatte intra- oder postoperativ eine (neue) neurologische Symptomatik. Dagegen waren 2 der 3 Patienten mit einem Verschluss in der Abstrombahn postoperativ (neu) symptomatisch.

\section{Retrospektive DSA-Zweit- beurteilung}

Zwei gegenüber intraoperativ zusätzlich detektierte hochgradige ACC-Stenosen waren im postoperativen Ultraschall $\mathrm{zu}$ einem asymptomatischen Frühverschluss (der ACI) progredient bzw. nicht 
Tab. 2 Ätiologie und Stenosierungsgrad der mittels intraoperativer DSA und postoperativer Duplexsonographie detektierten Auffälligkeiten bei 523 Patienten mit Thrombendarteriektomie einer A.-carotis-interna-Stenose

\begin{tabular}{|c|c|c|c|c|c|c|c|c|c|c|c|c|}
\hline & \multicolumn{9}{|c|}{ Operationsbereich lokal } & \multirow{2}{*}{\multicolumn{3}{|c|}{ Abstromgebiet }} \\
\hline & \multicolumn{3}{|c|}{ ACC } & \multicolumn{3}{|l|}{$\mathrm{ACl}$} & \multicolumn{3}{|l|}{ ACE } & & & \\
\hline & Vers & Hochgr & $<$ Hochgr & Vers & Hochgr & $<$ Hochgr & Vers & Hochgr & $<$ Hochgr & Vers & Hochgr & $<$ Hochgr \\
\hline DSA intraoperativ & 0 & 1 & 0 & 1 & 5 & 0 & 8 & 0 & 0 & 3 & 3 & 0 \\
\hline Ätiologie & - & $1 \mathrm{NA}$ & - & $1 \mathrm{TH}$ & $5 \mathrm{NA}$ & - & - & - & - & $3 \mathrm{TH}$ & $3 \mathrm{TH}$ & - \\
\hline DSA retrospektiv & 0 & $1+2$ & 0 & 1 & $5+3$ & 0 & 5 & 30 & 0 & $3+2$ & $3+3$ & 0 \\
\hline Ätiologie & - & $\begin{array}{l}1 \mathrm{NA}+ \\
2 \mathrm{TH}^{\mathrm{a}}\end{array}$ & - & $\mathrm{T}$ & $\begin{array}{l}5 \mathrm{NA}+ \\
3 \mathrm{UN}\end{array}$ & - & - & - & - & $\begin{array}{l}3 \mathrm{TH}+ \\
2 \mathrm{TH}^{\mathrm{b}}\end{array}$ & $\begin{array}{l}3 \mathrm{TH}+ \\
3 \mathrm{EN}^{\mathrm{c}}\end{array}$ & - \\
\hline $\begin{array}{l}\text { Duplexsonographie } \\
\text { postoperativ }\end{array}$ & 0 & 2 & 19 & 1 & 1 & 29 & 2 & 19 & 0 & 8 & $3+4$ & 0 \\
\hline Ätiologie & - & $2 \mathrm{IN}$ & $\begin{array}{l}16 \mathrm{IN} / \\
3 \mathrm{RE}\end{array}$ & $1 \mathrm{TH}^{\mathrm{a}}$ & $1 \mathrm{NA}$ & $\begin{array}{l}12 \mathrm{NA} / 14 \mathrm{KI} \\
2 \mathrm{HÄ} / 1 \mathrm{IN}\end{array}$ & - & - & A & $8 \mathrm{TH}^{\mathrm{bc}}$ & $\begin{array}{l}3 \mathrm{TH}+ \\
4 \mathrm{EN}^{\mathrm{b}}\end{array}$ & - \\
\hline $\begin{array}{l}\text { ACC A. carotis commu } \\
\text { IN Intima-Media-Läsio } \\
{ }^{a} \text { Eine intraoperative th } \\
{ }^{b} \text { Ein intraoperativ thro } \\
\text { 'Eine Engstellung zum }\end{array}$ & $\begin{array}{l}\text { nis, } A C l \\
\text { n, RE re } \\
\text { rombot } \\
\text { mbotisc }\end{array}$ & $\begin{array}{l}\text { A. carotis i } \\
\text { esidualer PI } \\
\text { tische ACC- } \\
\text { cher Versch }\end{array}$ & $\begin{array}{l}\text { erna, } A C E \text { A. } \\
\text { ue, KI Kinkin } \\
\text { enose zu AC } \\
\text { ss persistiert }\end{array}$ & $\begin{array}{l}\text { carotis ex } \\
\mathrm{g} / \text { Kaliber } \\
\text { I-Frühver } \\
\text { eine zu } \\
\text { ittiert }\end{array}$ & $\begin{array}{l}\text { xterna, } N A \\
\text { rsprung, } H \\
\text { rschluss } p \\
\text { hochgrad }\end{array}$ & $\begin{array}{l}\text { Nahteinziehung } \\
\text { perivaskuläres } \\
\text { gredient } \\
\text { en Stenose mi }\end{array}$ & $\begin{array}{l}\text { TH Thr } \\
\text { Hämatc } \\
\text { Engste }\end{array}$ & $\begin{array}{l}\text { rombus/Tr } \\
\text { om } \\
\text { ellung regr }\end{array}$ & $\begin{array}{l}\text { hrombembo } \\
\text { redient }\end{array}$ & lie, UN & annt, $E N$ & stellung, \\
\hline
\end{tabular}

mehr nachweisbar. Drei zusätzlich detektierte hochgradige ACI-Stenosen waren postoperativ ebenfalls nicht mehr nachweisbar und neurologisch nicht auffällig. In der Abstrombahn wurden zusätzlich zwei Verschlüsse detektiert, von denen einer symptomatisch war und persistierte, wohingegen der asymptomatische zu einer hochgradigen Stenose mit langstreckig enger distaler ACI partiell rekanalisierte. Zudem wurden in der Abstrombahn zusätzlich 3 hochgradige Stenosen in Form einer langstreckig engen distalen extrakraniellen ACI detektiert, von denen eine persistierte, eine remittierte und eine zum Verschluss progredient war, jeweils ohne neurologische Symptomatik.

\section{Postoperative Duplexsonographie}

Von den 523 Patienten erhielten 3 keine postoperative Duplexsonographie. Die 15 intraoperativ sofort Rekonstruierten wurden ebenfalls duplexsonographisch untersucht, gingen aber nicht in die vergleichende Auswertung zwischen Angiographie und Duplexsonographie ein, da eine Befunddiskrepanz nicht nur methodisch, sondern durch den zwischenzeitlich durchgeführten Korrektureingriff erklärt werden kann. Duplexsonographisch wurden in der ACC 18 IntimaMedia-Ablösungen (2 mit hochgradig stenosierendem Flap, eine davon symp- tomatisch) und 3 residuale Plaques detektiert, die angiographisch (intraoperativ/retrospektiv) nicht beschrieben wurden, darüber hinaus in der ACI am distalen Patchende 13 Nahteinziehungen (eine hochgradig stenosierend), 14 Kinking/Kalibersprünge am Übergang des Patches zum nichtoperierten Gefäßsegment, 2 extravasale Hämatome und eine Intima-Media-Ablösung, die alle nicht mit einer perioperativen Symptomatik assoziiert waren (• Tab. 2). In der Abstrombahn wurden gegenüber der Angiographie (intraoperativ/retrospektiv) zusätzlich 6 Verschlüsse (2 neu symptomatisch) und 2 hochgradige Stenosen in Form einer langstreckig engen extrakraniellen distalen ACI detektiert (jeweils asymptomatisch; • Tab. 2).

\section{DSA intraoperativ vs. retrospektiv vs. Duplex}

Die Sensitivität für intraoperativ aufgetretene Pathologien in Form hochgradiger Stenosen und Verschlüsse (ohne ACE-Pathologien) war für die beiden DSA-Auswertungen signifikant unterschiedlich ( $p=0,002$; McNemar-Test). Fünf zusätzliche hochgradige Stenosen lokal im Operationsbereich - durch einen Thrombus (2-mal in ACC) bzw. wahrscheinlich durch einen Thrombus (3-mal in ACI) - und 5 in der Abstrombahn (2 Thrombembolien und
3 langstreckig enge Arterien) fielen erst bei der retrospektiven Nachbeurteilung der intraoperativen DSA auf, womit sich die Rate gegenüber der intraoperativen Beurteilung von 2,5 auf $4,0 \%$ signifikant erhöhte. Die Häufigkeit beschriebener lokaler Pathologien nahm dabei von 1,3 auf 2,3\% zu, die von Pathologien in der Abstrombahn von 1,1 auf $2,1 \%$.

Der Vergleich aller drei Methoden erfolgte für jedwede lokale Pathologie der ACC und ACI (ohne ACE). Hierbei lag die Frequenz duplexsonographisch detektierter Pathologien signifikant über derjenigen, die in der DSA beschrieben worden waren $(10,1 \%$ vs. $2,3 \% ; p<0,001$, Chochran-Q-Test). Dies lag primär an der duplexsonographischen Detektion auch nicht okkludierender und nicht hochgradig stenosierender operativer Pathologien wie Intima-Media-Ablösungen in der ACC und Nahteinziehungen/ Kinkings/Kalibersprüngen am distalen Patchübergang zur ACI (๑ Tab. 2).

\section{Prädiktoren intraoperativer Pathologien}

Lokale Thrombosen und oder Thrombembolien in der Abstrombahn waren assoziiert mit notfallmäßigen Eingriffen im Rahmen eines akuten Schlaganfalls (adjustierte OR: 96,8; 95\%-KI: 11,4-819,7), höchstgradigen (>90\%) Stenosen (adjustierte OR: 5,8; $95 \%$-KI: 1,4-23,9) und 
einer fehlenden Thrombozytenaggregationshemmung präoperativ (adjustierte OR: 29,3; 95\%-KI: 1,9-442,6). Eine Assoziation mit lokalen Pathologien wie einer Intima-Media-Ablösung, einer Nahteinziehung und eines Kinkings/ Kalibersprungs bestand nicht.

Eine Nahteinziehung, ein Kinking und/oder ein Kalibersprung in der Regel am distalen Patchübergang zur normalen ACI waren assoziiert mit einer TEA der linken ACI (adjustierte OR: 2,4; $95 \%$-KI: $1,1-5,1)$ sowie der Operation ohne Patch (adjustierte OR: 16,6; $95 \%$ KI: 1,3-215,0) bzw. unter Verwendung eines Dacron-Patches (adjustierte OR: 3,0; $95 \%$-KI: 1,4-6,6), letztere jeweils gegenüber der Verwendung eines PTFEPatches.

\section{Intraoperative Pathologien und Outcome}

Keine der lokalen Pathologien - weder in ihrer Gesamtheit, noch im Hinblick auf spezifische Ätiologien oder bei Betrachtung nur relevanter Ausprägungen (Verschluss/hochgradige Stenose) - war unabhängig mit dem Outcome assoziiert. Dies traf nur für Abstrompathologien zu (adjustierte OR 5,3; KI 1,2-24,7) und hier speziell für Thrombembolien (adjustierte OR 5,7; KI 1,2-27,7), die neben dem Alter (adjustierte OR pro Jahr 1,18, KI 1,02-1,12), der Verwendung eines Shunts (adjustierte OR 3,3; KI 1,2-8,7) und einem notfallmäßigen TEA-Eingriff (adjustierte OR 9,8; KI 1,8-53,1) ein Prädiktor für einen perioperativen Schlaganfall und Tod waren. Dies änderte sich auch nach Ausschluss der Patienten mit einem notfallmäßigen TEA-Eingriff und Betrachtung nur der sekundärpräventiv operierten Patienten nicht.

\section{Diskussion}

In unserem Kollektiv wurden bei der Nachbeurteilung der Bilddokumentation der intraoperativen Kontroll-DSA 10 hochgradige Stenosen detektiert 5 lokal im Operationsbereich an ACC bzw. ACI und 5 in der Abstrombahn, die durch den Operateur bei der intraoperativen Beurteilung nicht beschrieben worden waren. Da alle 13 intraoperativ beschriebenen derartigen Pathologien auch in der Nachbeurteilung erkannt wurden, lag die Sensitivität der unmittelbaren Beurteilung durch den Operateur nur bei $57 \%$ und betrug die Rate detektierter derartiger Pathologien für die intraoperative DSA-Auswertung 2,5\% und für die retrospektive Auswertung $4,0 \%$. Dieser signifikante Unterschied scheint nicht alleine mit der spezifischen DSA-Durchführung und -Beurteilung in unserem Kollektiv zu erklären sein. So geben Studien, denen eine prospektive Auswertung der intraoperativen Kontrollangiographie zugrunde liegt [14, $18,23]$, für o.g. Pathologien Raten von $0,7-3,6 \%$ an, wohingegen diese bei retrospektiver Auswertung mit 6,1-10,4\% deutlich höher gefunden wurden [9, 20]. Sollten jedoch bis zu $40 \%$ der Verschlüsse und hochgradigen Stenosen an den unmittelbar hirnzuführenden Arterien intraoperativ durch den Operateur zum Zeitpunkt der Sofortrekonstruktionsmöglichkeit nicht erkannt werden, lässt dies am Nutzen einer routinemäßig durchgeführten intraoperativen DSA zweifeln und könnte einer der Gründe sein, weshalb bisher kein Zusammenhang zwischen Sofortrekonstruktion und Outcome gezeigt werden konnte.

A.-carotis-externa-Pathologien wurden bei diesem Vergleich nicht berücksichtigt. Deren Bedeutung hinsichtlich einer möglichen Sofortkorrektur ist gefäßchirurgisch umstritten [2], weshalb ihr Auftreten keine prinzipielle Indikation zu einem Reeingriff darstellte. Das Nichtbeschreiben einer ACE-Pathologie durch den Operateur schien uns daher nicht zwangsläufig auf deren Nichtvorliegen schließen zu lassen, sondern könnte auch Folge deren Einschätzung als irrelevant gewesen sein. Diese Fehlermöglichkeit schien uns auch beim Vergleich der Ergebnisse vorangegangener Studien mit den Ergebnissen unserer Studie für Verschlüsse und hochgradige Stenosen der unmittelbar hirnzuführenden Arterien am geringsten zu sein.

Bei allen angiographisch detektierten Pathologien, intraoperativ oder im Rahmen der retrospektiven Auswertung, handelte es sich um Verschlüsse oder hochgradige Stenosen. Dies war insbesondere für die retrospektive DSA-
Auswertung bemerkenswert, da diese formal und analog zur Auswertung der postoperativen Duplexsonographie erfolgte. Zwar lag die in unserer Untersuchung zur Anwendung kommende Kontrastmittel(KM)-Menge von 5-10 ml in der unteren Hälfte der in der Literatur beschriebenen $[9,15,17,18,20$, 22-24], die übrige technische Durchführung $\left(30^{\circ}\right.$-laterale als primäre, ggf. um weitere Projektionen ergänzte Aufnahme) war allerdings vergleichbar mit den meisten publizierten Studien. Auch macht der lange Beobachtungszeitraum mit Aktualisierung sowohl der angiographisch als auch duplexsonographisch zum Einsatz kommenden Geräte auf den jeweils zeitgemäßen Standard eine spezifische Gerätekonstellation als Ursache des Ergebnisses unwahrscheinlich.

Operativ induzierte hochgradige Stenosen und Verschlüsse sind für den betroffenen Patienten in Bezug auf eine Sofortrekonstruktion zwar die wichtigsten Befunde, geben aber dem Operateur vor dem Hintergrund der darüber hinaus zahlreichen duplexsonographisch detektierten operationstechnischen Unzulänglichkeiten keine für eine operationstechnische Qualitätskontrolle ausreichende Rückinformation. So wurden duplexsonographisch bei 10,3\% unserer Patienten im Operationsbereich an ACC oder ACI lokalisierte operationstechnische Unzulänglichkeiten beschrieben, was die angiographisch detektierte Rate von $2,3 \%$ weit übertrifft und in dieser Form auch von anderen Autoren gesehen wurde $[5,18]$ und eine methodische Schwäche der Angiographie in dieser Situation nahelegt.

Interessanterweise waren dabei die primär am distalen Übergang des Patches zum nicht desobliterierten Gefäßsegment gefundenen Nahteinziehungen und Kinkings/Kalibersprünge mit einer linksseitigen TEA assoziiert (ausschließlich rechtshändige Operateure), was für ein in dieser Konstellation schwierigeres operatives Prozedere spricht. Der ebenfalls gefundene Zusammenhang mit einer Operation unter Verwendung eines (einschichtigen) Dacron-Patches gegenüber eines (dreischichtigen) PTFEPatches könnte auf die geringere Steifig- 
keit des ersteren auf eine Fadenzugverformung zurückzuführen sein.

Den häufig beschriebenen Zusammenhang mit der Erfahrung des Operateurs konnten wir in unserem Kollektiv nicht nachweisen. Wir vermuten, dass dies zum einen statistische Gründe hat, so wurden knapp $80 \%$ der Operationen von erfahrenen und sehr erfahrenen Operateuren durchgeführt, und zum anderen an unserem Status eines akademischen Lehrkrankenhauses liegt. So wurde ein unerfahrener, sich in der gefäßchirurgischen Ausbildung befindender Operateur von vorn herein nicht als Erstoperateur für eine Operation eingeplant, die bereits präoperativ als möglicherweise technisch kompliziert eingeschätzt wurde.

Zwar kommt nicht allen duplexsonographisch detektierten Pathologien eine gleich hohe Relevanz zu, Intima-MediaAblösungen mitunter im Ausmaß eines Flaps, Nahteinziehungen und Kinkings/ Kalibersprünge am Übergang des Patches zum nicht desobliterierten Gefäßabschnitt können aber nicht per se als klinisch irrelevant angesehen werden und stellen zu vermeidende operative Unzulänglichkeiten dar. Auch der fehlende $\mathrm{Zu}$ sammenhang derartiger Pathologien mit dem klinisch-neurologischen Outcome in unserer Studie bzw. mit dem perioperativen Outcome allgemein $[4,9,17$, 23] erlaubt keine derartige Aussage, da hierbei nur Komplikationen bis 4 Wochen postoperativ eingehen. Dazu wäre eine longitudinale duplexsonographische und klinisch-neurologische Nachbeobachtung dieser Patienten erforderlich, die im Rahmen dieser Studie nicht erfolgten und bisher nur bei wenigen Untersuchungen zur intraoperativen Angiographie der Fall waren [10, 19], letztere mit zu wenigen Patienten, um auch eine mögliche Relevanz bei niedrigen Fallzahlen nachweisen zu können. Es überrascht daher nicht, dass wir in unserer Untersuchung "nur" für Abstrompathologien und hier speziell für Thrombembolien - neben „erwarteten“ Prädiktoren wie Alter, Shunt und notfallmäßigem TEAEingriff - einen Zusammenhang mit einem perioperativen Schlaganfall und Tod gefunden haben.
Der Vergleich zwischen Angiographie und Duplexsonographie schloss neben ACE-Pathologien (Begründung s. oben) auch Pathologien im Abstrombereich aus. Letztere haben in der Mehrzahl der Fälle andere als operationstechnische Ursachen. In unserem Kollektiv handelte es sich bei allen Abstrompathologien um Thrombembolien und langstreckig enge Arteriensegmente. Erstere zeigten keinen Zusammenhang mit lokalen operationstechnischen Unzulänglichkeiten wie Nahteinziehungen, Intima-Media-Ablösungen und Kinkings/Kalibersprüngen, sehr wohl aber mit der allgemeinen (Ausmaß der Thrombozytenaggregationshemmung, perakute Schlaganfallsituation) wie auch lokalen Thrombogenität (höchstgradige Stenose mit vermindertem poststenotischem Flussvolumen). Langstreckig enge Arteriensegmente - ätiologieunabhängig als „langstreckig enges Gefäße“ beschrieben - dürften ihre Ursache zum einen in bereits präoperativ distal einer höchstgradigen Stenose engen (aber nicht pseudookklusiv veränderten) Gefäßen haben wie auch in einem intraoperativ auftretenden Vasospasmus. Beide Pathologien (Thrombembolien, langstreckig enge Gefäße) variierten im Zeitraum zwischen intraoperativer DSA und postoperativer Duplexsonographie beträchtlich, sodass der methodische Vergleich unter Einbeziehung dieser Pathologien zur Fehleinschätzung der Validität der einzelnen Untersuchungsmethoden geführt hätte. Aus diesem Grund wurden auch die intraoperativ sofort rekonstruierten TEA vom $\mathrm{Me}$ thodenvergleich ausgeschlossen, da eine Befunddiskrepanz durch den zwischenzeitlich durchgeführten Korrektureingriff erklärt werden kann.

\section{Schlussfolgerung}

Die Ad-hoc-Beurteilung der intraoperativen Kontrollangiographie im Anschluss an eine TEA geht mit einer nicht unerheblichen, in unserem Kollektiv 40\% ausmachenden Rate an übersehenen hochgradigen Stenosen und Verschlüssen in den unmittelbar hirnzuführenden Arterien (ACC, ACI, intrakranielle ACI, MCA) einher. Dies stellt ihren routine- mäßigen Einsatz zur Erkennung sofort oder früh $\mathrm{zu}$ revidierender operativer Pathologien infrage und macht ihre alleinige Anwendung $\mathrm{zu}$ diesem Zweck obsolet. Die intraoperative DSA ist zur Detektion von i.d.R. nicht hochgradig stenosierenden operationstechnischen Unzulänglichkeiten methodisch nicht geeignet. Unabhängig von der Durchführung einer intraoperativen DSA sollte zur Qualitätskontrolle des operationstechnischen Ergebnisses postoperativ eine Duplexsonographie erfolgen, auch wenn die dabei detektierten operativen Unzulänglichkeiten wahrscheinlich mit einer sehr niedrigen Rate klinischneurologischer Komplikationen einhergehen.

\section{Korrespondenzadresse}

Prof. Dr. med. habil. Frank Meyer

Klinik für Allgemein-, Viszeral-, Gefäß- und

Transplantationschirurgie, Universitätsklinikum Magdeburg A.ö.R.

Leipziger Straße 44, 39120 Magdeburg,

Deutschland

frank.meyer@med.ovgu.de

Funding. Open Access funding enabled and organized by Projekt DEAL.

\section{Einhaltung ethischer Richtlinien}

Interessenkonflikt. J. Dillner, F. Meyer, Z. Halloul und M. Görtler geben an, dass kein Interessenkonflikt besteht.

Für diesen Beitrag wurden von den Autoren keine Studien an Tieren durchgeführt. Für die zugrundeliegende Studie am Menschen gelten die angegebene ethische Richtlinie und Vorgehensangaben.

Open Access. Dieser Artikel wird unter der Creative Commons Namensnennung 4.0 International Lizenz veröffentlicht, welche die Nutzung, Vervielfältigung, Bearbeitung, Verbreitung und Wiedergabe in jeglichem Medium und Format erlaubt, sofern Sie den/die ursprünglichen Autor(en) und die Quelle ordnungsgemäß nennen, einen Link zur Creative Commons Lizenz beifügen und angeben, ob Änderungen vorgenommen wurden.

Die in diesem Artikel enthaltenen Bilder und sonstiges Drittmaterial unterliegen ebenfalls der genannten Creative Commons Lizenz, sofern sich aus der Abbildungslegende nichts anderes ergibt. Sofern das betreffende Material nicht unter der genannten Creative Commons Lizenz steht und die betreffende Handlung nicht nach gesetzlichen Vorschriften erlaubt ist, ist für die oben aufgeführten Weiterverwendungen des Materials die Einwilligung des jeweiligen Rechteinhabers einzuholen. 
Weitere Details zur Lizenz entnehmen Sie bitte de Lizenzinformation auf http://creativecommons.org/ licenses/by/4.0/deed.de.

\section{Literatur}

1. Arning $C$, Widder $B$, von Reutern $G M$, Stiegler $H$, Görtler M (2010) Revision of DEGUM ultrasound criteria for grading intenal carotid artery stenoses and transfer to NASCET measurement. Ultraschal Med 31(3):251-257

2. Ascher E, Markevich N, Hingorani AP, Kallakuri S, Gunduz Y (2002) Internal carotid artery flow volume measurement and other intraoperative duplex scanning parameters as predictors of stroke after carotid endarterectomy. J Vasc Surg 35(1):439-444

3. Böckler D, Attigah N, Hakimi M, Allenberg JR (2009) ACST - Eine Studie wird zum Problem. ACST: how a trail can become a dilemma. Gefäßchirurgie 14(2):118

4. Courbier R, Jausseran JM, Reggi M, Bergeron $P$, Formichi M, Ferdani M (1986) Routine intraoperative carotid angiography: its impact on operative morbidity and carotid restenosis. J Vasc Surg 3(2):343-350

5. Donaldson MC, Ivarsson BL, Mannick JA, Whittemore AD (1993) Impact of completion angiography on operative conduct and results of carotid endarterectomy. Ann Surg 217(6):682-687

6. European Carotid Surgery Trialists' Collaborative Group (1991) MRC European Carotid Surgery Trial interim results for symptomatic patients with severe $(70-99 \%)$ or with mild $(0-29 \%)$ carotid stenosis. Lancet 337(8752):1235-1243

7. Executive Committee for the Asymptomatic Carotid Atherosclerosis Study (1995) Endarterectomy for asymptomatic carotid artery stenosis. JAMA 273(18):1421-1428

8. Kleiser B, Widder B (1992) Course of carotid artery occlusions with impaired cerebrovascular reactivity. Stroke 23(2):171-174

9. Lancelevee J, Maurel B, Gaudin M, Robin C, Bleuet $F$, Martinez $R$, Bertrand $P$, Lermusiauxet $P$ (2013) Is it mandatory to carry out completion arteriography after carotiendarterectomy with patch angioplasty? Ann Vasc Surg 27(6):719-726

10. Lipski DA, Bergamini TM, Garrison RN, Fulton RL (1996) Intraoperative duplex scanning reduces the incidence of residual stenosis after carotid endarterectomy. J Surg Res 60:317-320

11. MRC Asymptomatic Carotid Surgery Trial (ACST) Collaborative Group (2004) Prevention of disabling and fatal strokes by successful carotid endarterectomy in patients without recent neurological symptoms: randomised controlled trial. Lancet 363:1491-1502

12. National Institutes of Neurological Disorders and Stroke, Stroke and Trauma Division, North American Symptomatic Carotid Endarterectomy Trial (NASCET) investigators (1991) Clinical alert: benefit of carotid endarterectomy for patients with high-grade stenosis of the internal carotid artery. Stroke 22(6):816-817

13. Patresi C, Dorigo W, Troisi N, Fargion A, Innocenti AA, Pratesi G, Barbanti E, Pulliet R (2006) Routine completion angiography during carotid endarterectomy is not mandatory. Eur J Vasc Endovasc Surg 32(4):369-374

14. RiccoJB, de la Mothe GR, Fujita S, Page O, Valagier A Marchand C (2011) Impact of routine completion angiography on the results of primary carotid endarterectomy: a prospective study in a teaching hospital. Eur J Vasc Endovasc Surg 41(5):579-588

15. Ricco JB, Schneider F, Illuminati G (2013) Part one: for the motion. Completion angiography should be used routinely following carotid endarterectomy. Eur JVasc Endovasc Surg 45:416-419

16. Riles TS, Imparato AM, Jacobowitz GR, Lamparello PJ, Giangola G, Adelmann MA, Landis R (1994) The cause of perioperative stroke after carotid endarterectomy. J Vasc Surg 19(1):206-2016

17. Scott SM, Sethi GK, Bridgman AH (1982) Perioperative stroke during carotid endarterectomy: the value of intraoperative angiography. J Cardiovasc Surg 23(5):353-358

18. Valenti D, Gaggiano A, Berardi G, Ferri M, Mazzei R, Roda G, Palombo D (2003) Intraoperative Assessment of technical defects after carotid endarterectomy: a comparison between angiography and colour duplex scan. Cardiovasc Surg 11(1):26-29

19. Wallaert JB, Goodney PP, Vignati JJ, Stone DH, Nolan BW, Bertges DJ et al (2011) Completion imaging after carotid endarterectomy in the Vascular Study Group of New England. J Vasc Surg 42:144-152

20. Westerband A, Mills JL, Berman SS, Hunter GC (1997) The influence of routine completion arteriography on outcome following carotid endarterectomy. Ann Vasc Surg 11(1):14-19

21. Widder B, Paulat K, Hackspacher J, Mayr E (1986) Transcranial Doppler $\mathrm{CO}_{2}$ test for the detection of hemodynamically critical carotid artery stenosis and occlusions. Eur Arch Psychiatry Neurol Sci 236:162-168

22. Wieker CM, Harcos K, Ronellenfitsch U, Demirel S, Bruijnen H, Böckler D (2019) Impact of routine completion angiopgarphy on outcome after carotid endarterectomy. JVasc Surg 69(3):824-831

23. Woelfle KD, Bruijnen H, Neu J, Campbell P, Wack C, Loeprecht $H$ (2002) The role of intraoperative digital subtraction angiography for quality control of standard carotid endarterectomy using patch angioplasty. Cardiovasc Surg 10(2):116-122

24. Zannetti S, Cao P, De Rango P, Giordano G, Parlani G, Lenti M, Nora A (1999) Intraoperative Assessment of technical perfection in carotid endarterectomy: a prospective analysis of 1305 completion procedures. Eur J Vasc Endovasc Surg 18(1):52-58 
Hier steht eine Anzeige.

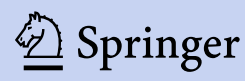

